\title{
REFERENCES
}

1. C. Berge, The theory of graphs and its applications, Wiley, New York, 1962.

2. R. Rado, Axiomatic treatment of rank in infinite sets, Canad. J. Math. 1 (1949), 337-343.

Marathon Oil Company, Littleton, Colorado

\section{CONCERNING CONTINUOUS IMAGES OF COMPACT ORDERED SPACES}

\section{B. TREYBIG ${ }^{1}$}

It is the purpose of this paper to prove that if each of $X$ and $Y$ is a compact Hausdorff space containing infinitely many points, and $X \times Y$ is the continuous image of a compact ordered space $L$, then both $X$ and $Y$ are metrizable. ${ }^{2}$ The preceding theorem is a generalization of a theorem [1] by Mardešić and Papić, who assume that $X, Y$, and $L$ are also connected. Young, in [3], shows that the Cartesian product of a "long" interval and a real interval is not the continuous image of any compact ordered space.

In this paper, the word compact is used in the "finite cover" sense. The phrase "ordered space" means a totally ordered topological space with the order topology. A subset $M$ of a topological space is said to be heriditarily separable provided each subset of $M$ is separable. If $a$ and $b$ are points of an ordered space $L$ and $a<b$, then $[a, b]$ $((a, b))$ will denote the set of all points $x$ of $L$ such that $a \leqq x \leqq b$ $(a<x<b)$, provided there is one; also, $[a, b]$ will be used even in the case where $a=b$. A subset $M$ of an ordered space $L$ is convex provided that if $a \in M, b \in M$, and $a<b$, then $[a, b] \subset M$. If $M$ is a subset of an ordered space $L$, then $G(M)$ will denote the set of all ordered pairs $(a, b)$ such that (1) $a \in M, b \in M$, and $a<b$, and (2) $\{a, b\}=M \cdot[a, b]$, provided there is one.

Lemma 0 . If $M$ is a compact subset of the ordered space $L$, then the relative topology of $L$ on $M$ is the same as the order topology on $M$.

Presented to the Society, May 4, 1962; received by the editors January 12, 1963 and, in revised form, July 3, 1963.

1 The author wishes to express his appreciation to the National Science Foundation for financial support.

${ }^{2}$ The referee has informed the author that the theorem of this paper was proved independently by A. J. Ward (Cambridge, England). 
LEMMA 1. If $M$ is a nondegenerate, totally disconnected, compact subset of an ordered space $L$, then $M$ is metrizable if and only if $G(M)$ is countable.

Proof. Suppose $M$ is metrizable. Since a compact Hausdorff space is metric if and only if it satisfies the second axiom of countability, there is a countable sequence $I_{1}, I_{2}, \cdots$ such that (1) for each $n$, $I_{n}$ is a convex open subset of $L$, and (2) $I_{1} \cdot M, I_{2} \cdot M, \cdots$ is a countable basis for $M$. There exists a transformation $T$ from $G(M)$ into the ordered pairs of positive integers such that if $(a, b) \in G(M)$ and $T((a, b))=(p, q)$, then $I_{p} \cdot\{a, b\}=a$ and $I_{q} \cdot\{a, b\}=b . T$ is easily seen to be a one-to-one transformation, so $G(M)$ is evidently countable.

Suppose $G(M)$ is countable. Let the elements of $G(M)$ be labeled $\left(a_{1}, b_{1}\right),\left(a_{2}, b_{2}\right), \cdots$. Let $H$ denote a collection such that $h \in H$ if and only if (1) there is a positive integer $i$ such that $h$ is the set of all points of $M$ which precede $b_{i}$, or $h$ is the set of all points of $M$ which follow $a_{i}$; or (2) there exist integers $i$ and $j$ such that $h=M \cdot\left[b_{i}, a_{j}\right]$. $H$ is a countable basis for $M$, so $M$ is metrizable.

Lemma 2. If $M$ is a separable subset of the ordered space $L$, then $M$ is hereditarily separable.

Proof. Suppose $H$ is a subset of $M$. There is a countable set $P_{1}, P_{2}, \cdots$ dense in $M$ such that if $P \in M$, then for some integer pair $(i, j), P_{i} \leqq P \leqq P_{j}$. For each integer pair $(i, j)$ such that $P_{i} \leqq P_{j}$ and $\left[P_{i}, P_{j}\right] \cdot H$ exists, let $H_{i j}$ denote a countable subset of $\left[P_{i}, P_{j}\right] \cdot H$ such that if $P \in\left[P_{i}, P_{j}\right] \cdot H$, then there exists $R$ in $H_{i j}$ and $S$ in $H_{i j}$ such that $R \leqq P \leqq S$. $\sum H_{i j}$ is easily seen to be a countable set dense in $H$.

LEMMA 3. If $M$ is a nonconnected, separable, compact subset of the ordered space $L$, then $M$ is metrizable if and only if $G(M)$ is countable.

LEMMA 4. If the continuous function $f_{1}$ maps the compact ordered space $K_{1}$ onto the Hausdorff space $S$, then there is a compact ordered space $K_{2}$ and a continuous function $f_{2}$ mapping $K_{2}$ onto $S$ such that (1) if $K$ is a closed proper subset of $K_{2}$, then $f_{2}(K) \neq S$, and (2) if $x$ and $y$ are elements of $K_{2}$ such that $f_{2}(x)=f_{2}(y)$, there is an element $z$ of $K_{2}$ between $x$ and $y$ such that $f_{2}(z) \neq f_{2}(x)$.

Proof. Let $H$ denote the set of closed subsets $m$ of $K$ such that $f_{1}(m)=S$. Define a partial order $\leqq$ on $H$ by saying $m_{1} \leqq m_{2}$ if and only if $m_{1} \subset m_{2}$. It is easily verified that each chain has a lower bound, so Zorn's lemma applies here, and $H$ has a minimal element $K$. 
For each point $x$ of $K$ let $K_{x}$ denote the union of all the subsets $k$ of $K$ such that (1) $x \in k$, (2) $k$ is convex relative to $K$, and (3) if $y \in k$, then $f_{1}(y)=f_{1}(x)$. Each $K_{x}$ is closed, and if $x$ and $y$ are elements of $K$, then either $K_{x}=K_{y}$ or $K_{x} \subset K-K_{y}$. Let $K_{2}$ denote the set of all $K_{x}$ for $x \in K$, and suppose $U$ is open in $K_{2}$ if and only if $U^{*}$ is open in $K .^{3}$ Suppose that $K_{2}$ is given the natural order induced by the order on $K$, and that $f_{2}$, which maps $K_{2}$ onto $S$, is defined by $f_{2}\left(K_{x}\right)=f_{1}(x)$. The space $K_{2}$ and the function $f_{2}$ satisfy the conclusion of the lemma.

LEMмA 5. If the continuous function $f$ maps the compact metric space $R$ onto the Hausdorff space $S$, then $S$ is metrizable.

Proof for the case Where $S$ is Nondegenerate. Let $R_{1}, R_{2}, \cdots$ denote a countable basis for $R$. Let $T$ denote a collection such that $U \in T$ if and only if there exists two points $s_{1}$ and $s_{2}$ of $S$ and a finite integer sequence $j_{1}, j_{2}, \cdots, j_{n}$ such that $f^{-1}\left(s_{1}\right) \subset \sum R_{j_{i}}, f^{-1}\left(s_{2}\right) \subset R$ $-\sum R_{j_{i}}$, and $U=S-f\left(R-\sum R_{j_{i}}\right)$. The collection $T$ is a countable basis for the compact Hausdorff space $S$, so $S$ is metrizable.

LEMMA 6. If the continuous function $f$ maps the compact ordered space $K$ onto the infinite, compact Hausdorff space $S$, then there exists a sequence $x_{0}, x_{1}, \cdots$ of distinct elements of $S$ such that $x_{1}, x_{2}, \cdots$ converges to $x_{0}$.

Proof. Let $y_{1}, y_{2}, \cdots$ denote a sequence of distinct elements of $S$, and for each $n$, let $z_{n}$ denote an element of $f^{-1}\left(y_{n}\right)$. There is an increasing sequence of integers $n_{1}, n_{2}, \cdots$ such that $z_{n_{1}}, z_{n_{2}}, \cdots$ is monotone, and since $K$ is compact, there is a point $z$ such that the latter sequence converges to $z$. There is a subsequence $j_{1}, j_{2}, \cdots$ of $n_{1}, n_{2}, \cdots$ such that $f\left(z_{j_{i}}\right) \neq f(z), i=1,2, \cdots$. The sequence $x_{0}, x_{1}, \cdots$ defined by $x_{0}=f(z)$ and $x_{i}=f\left(z_{j_{i}}\right), i \geqq 1$, satisfies the conclusion of the lemma.

Proof of theorem. Suppose that $X$ is not metrizable. Let $u$ denote an element of $X$, and let $g$ map $L$ continuously onto $X \times Y$. Since $\{u\} \times Y$ is the continuous image of a compact ordered space, an application of Lemma 6 yields an infinite sequence of distinct points $(u, b),\left(u, b_{1}\right),\left(u, b_{2}\right), \cdots$, all lying in $\{u\} \times Y$, such that $\left(u, b_{1}\right)$, $\left(u, b_{2}\right), \cdots$ converges to $(u, b)$. The space $Z=\left\{b, b_{1}, b_{2}, \cdots\right\}$ with the relative topology of $Y$ is an infinite compact Hausdorff space, and the space $X \times Z$ is the continuous image of a compact ordered space, so there exists a compact ordered space $K$ and a continuous function $f$ mapping $K$ onto $X \times Z$ such that the conclusions of Lemma 4 hold.

\footnotetext{
${ }^{3}$ If $U$ is a collection of point sets, then $U^{*}$ denotes the sum of the sets of the collection $U$.
} 
For each positive integer $n$, let $H_{n}$ denote a partition of $X \times Z$ into the following $n+1$ open and closed sets: $X \times\left\{b_{1}\right\}, X \times\left\{b_{2}\right\}, \cdots$, $X \times\left\{b_{n}\right\}, X \times\left\{b, b_{n+1}, b_{n+2}, \cdots\right\}$. For each $n$, let $K_{n}$ denote the set of all $f^{-1}(h)$ for $h \in H_{n}$, and let $I_{n}$ denote a partition of $K$ into convex open and closed sets such that if $I \in I_{n}$, there is an element $k$ of $K_{n}$ such that $I \subset k$. Since $K$ is compact, each $I_{n}$ is a finite collection. Let $C$ denote a point set to which a point $P$ belongs if and only if there is an integer $n$ and an element $I$ of $I_{n}$ which intersects $f^{-1}(X \times\{b\})$ such that $P$ is either the right-most point of this intersection or the left-most. $C$ is a countable set which will be shown to be dense in $f^{-1}(X \times\{b\})$.

Suppose the set $f^{-1}(X \times\{b\})$ contains an open set $U$. Let $P$ denote an arbitrary point of $f^{-1}(X \times\{b\})$ and suppose $f(P)=(x, b)$. For each $n$ let $Q_{n}$ denote an element of $f^{-1}\left(x, b_{n}\right)$. Some subsequence of the $Q_{i}$ 's converges to a point $Q$ in $K-U$, and the continuity of $f$ implies that $f(Q)=(x, b)$. Therefore, $f(K-U)=X \times Z$, which is a contradiction. Now suppose that $P \in f^{-1}(X \times\{b\})$ and $R<P<S$. There is a positive integer $n$ and a point $Q$ of $f^{-1}\left(X \times\left\{b_{n}\right\}\right)$ in $(R, S)$. Suppose $P<Q<S$. There is an element $I$ of $I_{n}$ containing $P$, but not $Q$, and the rightmost point $T$ of $I \cdot f^{-1}(X \times\{b\})$ is an element of $C$ satisfying $P \leqq T$ $<S$. This case clearly shows why $C$ is dense in $f^{-1}(X \times\{b\})$.

The separability of $f^{-1}(X \times\{b\})$ implies that $X \times\{b\}$ is separable, and consequently, that $X \times Z$ is separable. Let $\left\{R_{1}, R_{2}, \cdots\right\}$ denote a countable set dense in $X \times Z$, and for each $n$ let $P_{n}$ denote an element of $f^{-1}\left(R_{n}\right)$. The set $K^{\prime}=\operatorname{cl}\left(\sum P_{i}\right)$ is a closed subset of $K$ such that $f\left(K^{\prime}\right)=X \times Z$, so $K^{\prime}-K$ and $K$ is separable.

It will now be shown that $X \times Z$ satisfies the first axiom of countability. ${ }^{4}$ Let $P$ denote an arbitrary point of $X \times Z$. Since $f^{-1}(P)$ is compact and $K-f^{-1}(P)$ is separable, it follows by an easy argument that there is a countable set $\left\{Q_{1}, Q_{2}, \cdots\right\}$ dense in $K-f^{-1}(P)$ such that if $x \in f^{-1}(P)$ and $y \in K-f^{-1}(P)$, there is a $Q_{i}$ such that $x<Q_{i} \leqq y$ or $y \leqq Q_{i}<x$. For each positive integer $n$, let $V_{n}$ denote a collection to which $v$ belongs if and only if there is a point $z$ of $f^{-1}(P)$ such that $v$ is the maximal convex subset of $K$ which contains $z$ and does not intersect $\sum_{1}^{n} Q_{i}$. Since, for each $n, V_{n}^{*}$ is an open subset of $K$ containing $f^{-1}(P)$, it follows that the set $T_{n}=X \times Z-f\left(K-V_{n}^{*}\right)$ is an open subset of $X \times Z$ containing $P$. Suppose $Q$ is an arbitrary point of $X \times Z$ distinct from $P$, that $z \in f^{-1}(Q)$, and also, for example, that $z_{1}$ is the last point of $f^{-1}(P)$ which precedes $z$ and $z_{2}$ is the first point of $f^{-1}(P)$ which follows $z$. There exist an integer $j_{1}$ and an integer $j_{2}$

\footnotetext{
- It also may be shown from [2] that $X \times Z$ satisfies the first axiom of countability.
} 
such that $z_{1}<Q_{j_{1}} \leqq z \leqq Q_{j_{2}}<z_{2}$. The set $V_{j}^{*}$, where $j=\max \left(j_{1}, j_{2}\right)$, does not contain $z$, so the set $T_{j}$ does not contain $Q$. Therefore, $T_{1}, T_{2}, \cdots$ is a countable sequence of open sets having only $P$ in common.

The set $f^{-1}\left(X \times\left\{b_{1}\right\}\right)$ is not metrizable, since that would imply that $X$ is metrizable. Since Lemma 2 implies that $f^{-1}\left(X \times\left\{b_{1}\right\}\right)$ is separable, Lemma 3 implies that $G_{1}=G\left(f^{-1}\left(X \times\left\{b_{1}\right\}\right)\right)$ is uncountable. There does not exist an uncountable subcollection $U_{1}$ of $G_{1}$ such that if $(x, y) \in U_{1}$, then $f(x)=f(y)$; for if there does, the conditions on $f$ imply that for $(x, y) \in U_{1}$, there is a $P_{i}$ such that $x<P_{i}<y$, which is a contradiction. Suppose there is an uncountable subcollection $U_{2}$ of $G_{1}$ and a point $x$ of $X$ such that if $(z, w) \in U_{2}$, then $f(z)=\left(x, b_{1}\right)$ or $f(w)=\left(x, b_{1}\right)$. There is an uncountable subcollection $U_{3}$ of $U_{2}$ such that if $(z, w) \in U_{3}$ and $f(z)=\left(x, b_{1}\right)$, then $f(w) \neq\left(x, b_{1}\right)$. The fact that $X \times Z$ has a countable basis at $\left(x, b_{1}\right)$ implies that there is an open set $U$ containing $\left(x, b_{1}\right)$ and an uncountable subcollection $U_{4}$ of $U_{3}$ such that if $(z, w) \in U_{4}$ and $f(z) \in U$, then $f(w) \in(X \times Z)-U$. There is a point $t$ of $K$ such that each open set containing $t$ contains uncountably many elements $(z, w)$ of $U_{4}$. The continuity of $f$ would imply that $f(t)=\left(x, b_{1}\right)$ and that $f(t) \in(X \times Z)-U$, which is a contradiction.

Let $C$ denote the collection of all subsets $M$ of $G_{1}$ such that if $(p, q)$ and $\left(p^{\prime}, q^{\prime}\right)$ are elements of $M$ then $f(p), f(q), f\left(p^{\prime}\right)$, and $f\left(q^{\prime}\right)$ are four distinct points. $C$ is partially ordered by inclusion, and each chain has an upper bound, so Zorn's lemma implies the existence of a maximal element $W$. Suppose $W$ is countable. Let $D$ denote the set of all elements $(p, q)$ of $G_{1}$ such that there is an element $\left(p^{\prime}, q^{\prime}\right)$ of $W$ such that $f(p)=f\left(p^{\prime}\right)$ or $f\left(q^{\prime}\right)$, or $f(q)=f\left(p^{\prime}\right)$ or $f\left(q^{\prime}\right)$. D is countable, so there is an element $(p, q)$ of $G_{1}-D$ such that $f(p) \neq f(q)$. However, $W+\{(p, q)\}$ is an element of $C$ containing $W$, so $W$ is not maximal. This is a contradiction, so $W$ is uncountable.

It will now be shown that if $x_{1}$ and $x_{2}$ are points in $X$, then there is a positive integer $N$ such that if $n>N, z_{1} \in f^{-1}\left(x_{1}, b_{n}\right)$, and $z_{2} \in f^{-1}\left(x_{2}, b_{n}\right)$, then there is a point of $K$ between $z_{1}$ and $z_{2}$. On the contrary, suppose there exist points $x_{1}$ and $x_{2}$ of $X$ and an increasing sequence of integers $n_{1}, n_{2}, \cdots$ such that for each $i$ there exist points $z_{i}$ and $w_{i}$ of $f^{-1}\left(x_{1}, b_{n_{i}}\right)$ and $f^{-1}\left(x_{2}, b_{n_{i}}\right)$, respectively, such that no point of $K$ lies between $z_{i}$ and $w_{i}$. There is a point $z$ of $K$ such that each open set about $z$ contains, for infinitely many integers $i$, both $z_{i}$ and $w_{i}$. But the continuity of $f$ would imply that $f(z)=\left(x_{1}, b\right)$ and also that $f(z)=\left(x_{2}, b\right)$, which is a contradiction.

Let $V$ denote the set of all ordered pairs $(x, y)$ such that there is an element $(z, w)$ of $W$ such that $f(z)=\left(x, b_{1}\right)$ and $f(w)=\left(y, b_{1}\right)$. There is a positive integer $N$ and an uncountable subcollection $V_{1}$ of $V$ such that if $(x, y) \in V_{1}, z \in f^{-1}\left(x, b_{N}\right)$, and $w \in f^{-1}\left(y, b_{N}\right)$, then there 
is some point of $K$ between $z$ and $w$. Let $T_{1}$ denote a set to which $t$ belongs if and only if there exist integers $i$ and $j$ such that $t$ is maximal with respect to the property of being a convex subset of $K$ which contains neither $P_{i}$ nor $P_{j}$. Let $T_{2}$ denote a collection to which $t$ belongs if and only if $t \in T_{1}$ or $t$ is the union of a finite number of elements of $T_{1}$. The collection $T_{2}$ is countable and has the property that if $(x, y) \in V_{1}$ then there exist elements $t_{1}$ and $t_{2}$ of $T_{2}$ such that $f^{-1}\left(x, b_{N}\right)$ $\subset t_{1} \subset K-f^{-1}\left(y, b_{N}\right)$ and $f^{-1}\left(y, b_{N}\right) \subset t_{2} \subset K-f^{-1}\left(x, b_{N}\right)$. This is easily seen, because for each $z$ in $f^{-1}\left(x, b_{N}\right)$, for example, there is an element $t_{z}$ of $T_{1}$ which contains $z$ and does not intersect $f^{-1}\left(y, b_{N}\right)$, and $f^{-1}\left(x, b_{N}\right)$ is covered by a finite number of the $t_{z}$ 's.

Let $S_{1}$ denote a collection to which an element $s$ belongs if and only if there is an element $t$ of $T_{2}$ such that $\left(s \times\left\{b_{N}\right\}\right)=X \times\left\{b_{N}\right\}$ $-f(K-t) \cdot\left(X \times\left\{b_{N}\right\}\right) . S_{1}$ is a countable collection of open subsets of $X$ such that if $(x, y) \in V_{1}$, there exist elements $s_{1}$ and $s_{2}$ of $S_{1}$ such that $x \in s_{1} \subset X-\{y\}$ and $y \in s_{2} \subset X-\{x\}$. Since $S_{1}$ is countable and $V_{1}$ is uncountable, there is an element $s$ of $S_{1}$ and an uncountable subcollection $V_{2}$ of $V_{1}$ such that if $(x, y) \in V_{2}$, then $x \in s \subset X-\{y\}$. Since $f$ is continuous and $s \times\left\{b_{1}\right\}$ is open in $X \times Z$, it follows that $f^{-1}\left(s \times\left\{b_{1}\right\}\right)$ is open in $K$.

Let $W_{1}$ denote the collection of all elements $(c, d)$ of $W$ such that there is an element $(x, y)$ of $V_{2}$ such that $(f(c) ; f(d))=\left(x, b_{1} ; y, b_{1}\right)$. If $(c, d) \in W_{1}, c \in f^{-1}\left(s \times\left\{b_{1}\right\}\right)$ and $d \in K-f^{-1}\left(s \times\left\{b_{1}\right\}\right)$. For each pair $(c, d)$ of $W_{1}$ let $U(c)$ denote a convex open subset of $K$ such that $c \in U(c)$ and $U(c) \subset f^{-1}\left(s \times\left\{b_{1}\right\}\right)$. The set of all $U(c)$ 's is uncountable and no two of them intersect, so $K$ is not separable. This yields a contradiction, so $X$ is metrizable.

One interesting application of the preceding theorem is the following

THEOREM. If a space $X$ is the continuous image of a compact ordered space and can be expressed as an infinite product $\left(\Pi X_{i}\right)$, where each $X_{i}$ is a nondegenerate compact Hausdorff space, then (1) the product is a countable product, and (2) each $X_{i}$ is metrizable.

\section{REFERENCES}

1. S. Mardesiic and P. Papić, Continuous images of ordered continua, Glasnik Mat.-Fiz. Astronom. 15 (1960), 171-178.

2. - Diadic bicompacta and continuous mappings of ordered bicompacta, Dokl. Akad. Nauk SSSR 143 (1962), 529-531. (Russian)

3. G. S. Young, Representations of Banach spaces, Proc. Amer. Math. Soc. 13 (1962), 667-668.

Tulane University 